\title{
ATIVIDADE IN VITRO DO EXTRATO HIDROALCOÓLICO DE LIPPIA SIDOIDES CHAM SOBRE LARVAS DE TERCEIRO ESTÁDIO DE NEMATÓDEOS GASTRINTESTINAIS (FAMÍLIA TRICHOSTRONGYLIDAE) DE CAPRINOS
}

\author{
W.M.A. Souza ${ }^{1 *}$, R.A. Ramos $^{1}$, I.C.B. Silva ${ }^{2}$, L.C. Alves ${ }^{1}$, M.C.O.C.C. Coelho ${ }^{1}$, M.B.S. Maia ${ }^{3}$ \\ ${ }^{1}$ Universidade Federal Rural de Pernambuco, Departamento de Medicina Veterinária, Av. D. Manoel \\ Medeiros, s/nº, CEP 52171-900, Recife, PE, Brasil. E-mail: isabelwagner@uol.com.br
}

\section{RESUMO}

\begin{abstract}
Avaliou-se in vitro a atividade larvicida do extrato hidroalcóolico de alecrim pimenta (Lippia sidoides Cham) sobre larvas de terceiro estádio de nematoides gastrintestinais de caprinos. Os resultados encontrados demonstraram que a concentração de $500 \mathrm{mg} \mathrm{mL}^{-1}$ apresentou atividade efetiva e com capacidade de ação de $95,89 \%$, demonstrando desta forma a possibilidade do uso terapêutico anti-helmíntico para esse extrato.
\end{abstract}

PALAVRAS-CHAVE: Fitoterapia, parasitoses, anti-helmíntico, alecrim pimenta.

\section{ABSTRACT}

IN VITRO ACTIVITY OF THE HYDROALCOHOLIC EXTRACT OF LIPPIA SIDOIDES CHAM ON BUOYANT LARVAE OF THIRD-STAGE GASTROINTESTINAL NEMATODES (FAMILY TRICHOSTRONGYLIDAE) OF GOATS. The in vitro larvicidal activity of the hydroalcoholic extract of rosemary pepper (Lippia sidoides Cham) was evaluated on buoyant larvae of third-stage gastrointestinal gastrointestinal nematodes of goats. The results demonstrated that the concentration of $500 \mathrm{mg} \mathrm{mL}^{-1}$ presented effective activity, with a capacity of action of $95.89 \%$, thus demonstrating the possibility of anthelmintic therapeutic use for this extract.

\section{KEY WORDS: Phytotherapy, anthelmintic, rosemary pepper.}

A prática da medicina popular com o uso de plantas é bastante antiga, não só no Brasil como no mundo, sendo atualmente importante na cura e na prevenção de eventuais enfermidades. Por ser um campo bastante amplo, existindo milhares de espécies distintas com propriedades terapêuticas e diferentes formas de uso para cada grupo, pesquisas estão sendo feitas em todo o país visando contribuir para um melhor aproveitamento dessa flora, em virtude da sua enorme aplicabilidade (AlCÂNTARA JUNIOR et al., 2005).

Os parasitos gastrintestinais dos ruminantes são responsáveis por constantes perdas econômicas na caprinovinocultura do Nordeste do Brasil (PINHEIRO et al., 2000). Dentre os nematoides, o Haemonchus contortus (família Trichostrongylidae) é responsável por elevada mortalidade e diminuição da produtividade dos animais, particularmente nos mais jovens (Arosemena et al., 1998).

Ocontrole dos parasitos gastrintestinais tem sido realizado por meio de dosificação anti-helmíntica, visando reduzir os níveis de infecção dos animais e, consequentemente, a descontaminação das pastagens (CHARLes, 1989). Porém, o desenvolvimento de resistência aos anti-helmínticos, associado ao alto custo do tratamento, seus resíduos em alimentos e a poluição ambiental causada por sua utilização, tem incentivado a pesquisa de terapêutica alternativa, como os fitoterápicos (HERD, 1996). Muitas plantas já têm sido descritas como possuidoras de atividade anti-helmíntica (HAMmond et al., 1997; VIEIRA; CAVALCANTI, 1999).

A Lippia sidoides Cham pertence a um gênero bastante vasto, sendo composto por aproximadamente 200 espécies de ervas, arbustos e pequenas árvores pertencentes à família Verbenaceae (TERblanchÉ; Kornelius, 1996). No óleo essencial de L. sidoides encontram-se vários princípios químicos diferentes, destacando-se: monoterpernos (carvacrol, p-cimeno, timol, a-felandreno), sesquiterpene ( $\beta$-cariofileno, a-copaeno, a-humuleno) (MACAMBIRA et al., 1986; Lemos et al., 1990; TerblanChÉ; Kornelius, 1996).

${ }^{2}$ Médica Veterinária, Jaboatão dos Guararapes, PE, Brasil.

${ }^{3}$ Universidade Federal Rural de Pernambuco, Departamento de Fisiologia e Farmacologia, Recife, PE, Brasil.

*Programa de pós-graduação em Ciência Veterinária (Doutorado) - UFRPE. 
A associação destes compostos promove ao óleo essencial da L. sidoides forte ação antifúngica, antibacteriana, antimicrobiana, moluscida e larvicida, respectivamente, contra Staphylococcus aureus, Streptococcus mutans, Corynebacterium xerosis, Candida albicans ou Monilia, Trichophytum rubrume T. interdigitale, Biomphalaria glabra e Aedes aegypti (LEMOS et al., 1990; LACOSTE et al., 1996; Matos et al., 2000; LORENZI; MATOS, 2002).

Devido ao potencial terapêutico apresentado pela L. sidoides, sua alta distribuição pela flora nordestina e a necessidade de se encontrar medidas alternativas eficazes no controle dos parasitos gastrintestinais dos caprinos e ovinos, objetivou-se avaliar a açãolarvicida do extrato hidroalcoólico seco (EHA) de L. sidoides sobre larvas infectantes de nematódeos gastrintestinais (família Trichostrongylidae) de Caprinos.

Foram utilizadas folhas de L. sidoides Cham provenientes da região do semiárido pernambucano $\left(8^{\circ} 25^{\prime} 00^{\prime \prime}\right.$ Sul e $37^{\circ} 03^{\prime} 15^{\prime \prime}$ Oeste) e transportadas ao laboratóriode produtos bioativos de Departamentode Fisiologia e Farmacologia, da Faculdade de Farmácia da Universidade Federal de Pernambuco onde o extrato vegetal foi obtido. Inicialmente as folhas foram trituradas e desidratadas em estufa durante 1 hora a $50^{\circ} \mathrm{C}$. Em seguida foi adicionado Etanol $/ \mathrm{H}_{2} \mathrm{O} 70^{\circ}$ GL (1:1), e procedida a homogeneização e incubação em banho-maria a $70^{\circ} \mathrm{C}$ por $1 \mathrm{~h}$ e $30 \mathrm{~min}$. Após este período, foi realizada a filtração em funil com algodão e o extrato obtido foi concentrado em rota evaporador (com pressão reduzida à temperatura de $50^{\circ} \mathrm{C} 90$ rpm), A L. sidoides Cham foi identificada e tombada no Herbário IPA-PE sob no 82505 pela botânica Olívia Cano, funcionária da mesma instituição.

Foram coletadas $10 \mathrm{~g}$ de fezes diretamente da ampola retal de 20 animais da espécie caprina criados em sistema semi-intensivo no Município de Jaboatão dos Guararapes ( $8^{\circ} 10^{\prime} 00^{\prime \prime}$ Sul e $35^{\circ} 08^{\prime} 00^{\prime \prime}$ Oeste), Estado de Pernambuco. As fezes foram acondicionadas em sacos plásticos e encaminhadas sob temperatura refrigerada ao Laboratório de Doenças Parasitárias dos Animais Domésticos da Universidade Federal Rural de Pernambuco.

Foi realizada cultura para obtenção das larvas infectantes, incubadas em temperatura média de $26^{\circ} \mathrm{C}$ duranteoitodias, reviradase recolhidas cincoamostras contendo $50 \mu \mathrm{L}$ do líquido. As larvas obtidas foram coradas com Lugol 1\%, realizando-se a classificação quanto ao gênero segundo UENO; GonçALVES (1998). Foi retirado novamente um número igual de amostras para avaliação de larvas vivas e a sua motilidade.

Microplacas de poliestireno com fundo redondo de 96 poços, em triplicata, contendo $50 \mu \mathrm{L}$ da solução com aproximadamente 100 larvas $\mathrm{L}_{3}$ nas contrações $\mu$ controle água, $\alpha 21,0 \mathrm{mg} / \mathrm{mL}, \alpha 32,0 \mathrm{mg} / \mathrm{mL}, \alpha 4$ $5,0 \mathrm{mg} / \mathrm{mL}$, $\alpha 510 \mathrm{mg} / \mathrm{mL}, \alpha 620 \mathrm{mg} / \mathrm{mL}, \alpha 750$ $\mathrm{mg} / \mathrm{mL}, \alpha 8100 \mathrm{mg} / \mathrm{mL}, \alpha 9150 \mathrm{mg} / \mathrm{mL}, \alpha 10250$ $\mathrm{mg} / \mathrm{mL}$ e $\alpha 11500 \mathrm{mg} / \mathrm{mL}$, do extrato vegetal, $\alpha 12$ controle febendazole a $33 \mathrm{mg} / \mathrm{mL}$ foram incubados em temperatura ambiente acompanhada a $26^{\circ} \mathrm{C}$ por 24,48 e 72 horas. Sendo o material retirado após cada período de tempo, colocados em lâminas de vidro e analisado em microscopia ótica com objetiva de100x, sendo as larvas contadas e avaliadas quanto a sua viabilidade. Oprocedimento foi igualmente repetido comágua destilada (controlenegativo) eFebendazole a $33 \mathrm{mg} / \mathrm{mL}$ (controle positivo) constituindo-se os grupos controles para os testes.

Foramaplicados métodosa fim demensurara eficácia do EHA. A fim de alcançar o objetivo desta parte da análise, calculou-se o percentual de larvas mortas após 24, 48 e 72 horas para cada tratamento. Modelo de efeito dada por $Y_{\mathrm{ijk}}=\mu+\alpha_{\mathrm{i}}+\beta_{\mathrm{j}}+\alpha \beta_{\mathrm{ij}}+\varepsilon_{\mathrm{ijk}}$.

Verificou-se, por meio do teste de Lilliefors (STEPHENS, 1974), se a suposição de normalidade dos resíduos era satisfeita, ou seja, $\varepsilon_{\mathrm{ij}} \sim \mathrm{N}\left(0, \sigma^{2}\right)$. Calculouse a análise de variância para verificar se o efeito tratamento, efeito horas e/ ou efeito interação (tratamento $x$ horas) eram significativos para determinar o percentual de larvas mortas pelo extrato utilizado. Para os fatores que foram significativos houve comparação entre as médias para verificar onde os efeitos eram maiores ao utilizar extrato nas $\mathrm{L}_{3}$.

Os dados foram tabulados na planilha eletrônica Microsoft Excel. Para análise estatística foram utilizados os softwares R versão 2.2.1 e EPI6. Todas as decisões foram tiradas no nível de significância de $5 \%$.

A cultura das larvas de nematoides gastrintestinais da família Trichostrongylidae na espécie caprina revelou larvas infectantes do gênero Haemonchus, Trichostrongylus e Oesophagostomum, com uma predominância do gênero Haemonchus estando de acordo com Almeida et al. (2003) ao relatarem a predominância do gênero Haemonchus em relação aos outros em ovinos no Nordeste do Brasil.

Por meio das estimativas do modelo de efeito, verificou-se que os coeficientes referentes ao tempo (horas) e a interação com as diferentes concentrações não foram significativos. Contudo, por meio da análise de variância do modelo de efeito final, observa-se que o efeito do tratamento é significativo (Tabela 1).Sendoassim, foi realizado o teste de Tukey a fim de verificar onde existe maior ação do extrato.

$\mathrm{O}$ grupo a11 apresentou o melhor resultado quando comparado a todos os outros grupos testados, sendo observados 95,89\% de eficácia. Como indicativo da eficiência foi considerada o que preconiza o Grupo Mercado Comum para substâncias químicas: Altamente efetivo > 98\%; Efetivo 90-98\%; Moderadamente efetivo 80-89\% e Insuficientemente ativo $<80 \%$ (GMC, 1996).

Sendo assim, observa-se, com os resultados expostos, que o efeito do extrato é dose dependente. À medida queaumenta a concentração, aumenta o efeito. 
Tabela 1 - Análise de variância para o modelo de efeito final.

\begin{tabular}{lccccc}
\hline Fonte de variação & Graus de liberdade & Soma de quadrados & Quadrado médio & F & p-valor \\
\hline$\alpha$ & 11 & 41152 & 3741 & 54,972 & $<0,001^{1}$ \\
Resíduo & 72 & 5604 & 78 & - & - \\
\hline
\end{tabular}

${ }^{1}$ P-valor do teste do teste F. Se p-valor $<0,05$.

O grupo controle positivo $\alpha 12$ (febendazole $33 \mathrm{mg}$ / $\mathrm{mL}$ ) apresentou valores de referência semelhantes aos grupos $\alpha 7$, $\alpha 8$ e $\alpha 9$ com média positiva, porém não significativa. $\mathrm{O}$ que nos revela que este grupo também se encontra abaixo do controle efetivo de larvas, o que possivelmente pode demonstrar o desenvolvimento da resistência anti-helmíntica ao produto febendazole. Uma vez que este princípio ativo é indicado com a ação larvicida segundo o fabricante. Quando comparado aos grupos $\alpha 10$ e $\alpha 11$, o febendazole se encontra com valores abaixo desses, porém bem próximo ao $\alpha 10$ e não significativo, ficando desta forma bem inferior ao grupo $\alpha 11$.

Os resultados aqui encontrados estabelecem uma relação semelhante dos grupos $\alpha 10$ e $\alpha 11$ com os dados deBEVILAQUAetal.(2005) ao estudaremoefeitolarvicida do óleo essencial de L. sidoides sobre as larvas $\mathrm{L}_{3}$ de $H$. contortus nas concentrações $2 \%$ e $1 \%$ com resultados de $94,58 \%$ e 90, 25\%, respectivamente, de eficiência.

BEvilaqua et al. (2005), ao avaliarem a ação larvicida do óleo essencial de Ossimum gratissimum, puderam observar uma alternância de eficiência nos resultados encontrados, mesmo com o aumento de suas concentrações $0,125 \%$ obteve $41,18 \%$ e $0,25 \%$, $0,50 \% 1 \%$ e $2 \%$ obtiveram respectivamente $45,78 \%$, $41,98 \%, 76,51 \%$ e $93,22 \%$ de ação. Esse mesmo efeito também foi observado neste estudo entre os grupos
T2 a T6, mas sem diferenças significativas havendo apenas a alternância dos resultados.

Essa situação pode ser explicada quando, durante o desenvolvimento da metodologia, observou-seque quando o volume do extrato era menor, o volume final também diminuía e poderia haver um efeito de aumento da densidade final, interferindo de alguma forma no resultado.

A possibilidade de variação de resultados foi observada por HounZANGBe-Adote et al. (2005) que estudaram a ação de quatro plantas tropicais Zanthoxylum zanthoxyloides, Newbouldia laevis, Morinda lúcida e Carica papaya em ovos, larvas infectantes e adultos de $H$. contortus verificando que as plantas testadas apresentavam ação contra todos os estágios dos parasitos, porém, variando grandemente seus resultados entre cada estágio. Desta forma, a discussão de trabalhos referentes a esses estudos é complexa, pois se trata de material, concentrações e solventes diferentes e, portanto, apresentando possivelmente reação para os estágios de desenvolvimento do parasito próprio.

A concentração de $500 \mathrm{mg} / \mathrm{mL}$ do EHA de Lippia sidoide Cham demonstrou in vitro ação larvicida com 95,89\% de eficiência sobre as larvas $\mathrm{L}_{3}$ de nematoides gastrintestinais (família Trichostrongylidae) de caprinos.

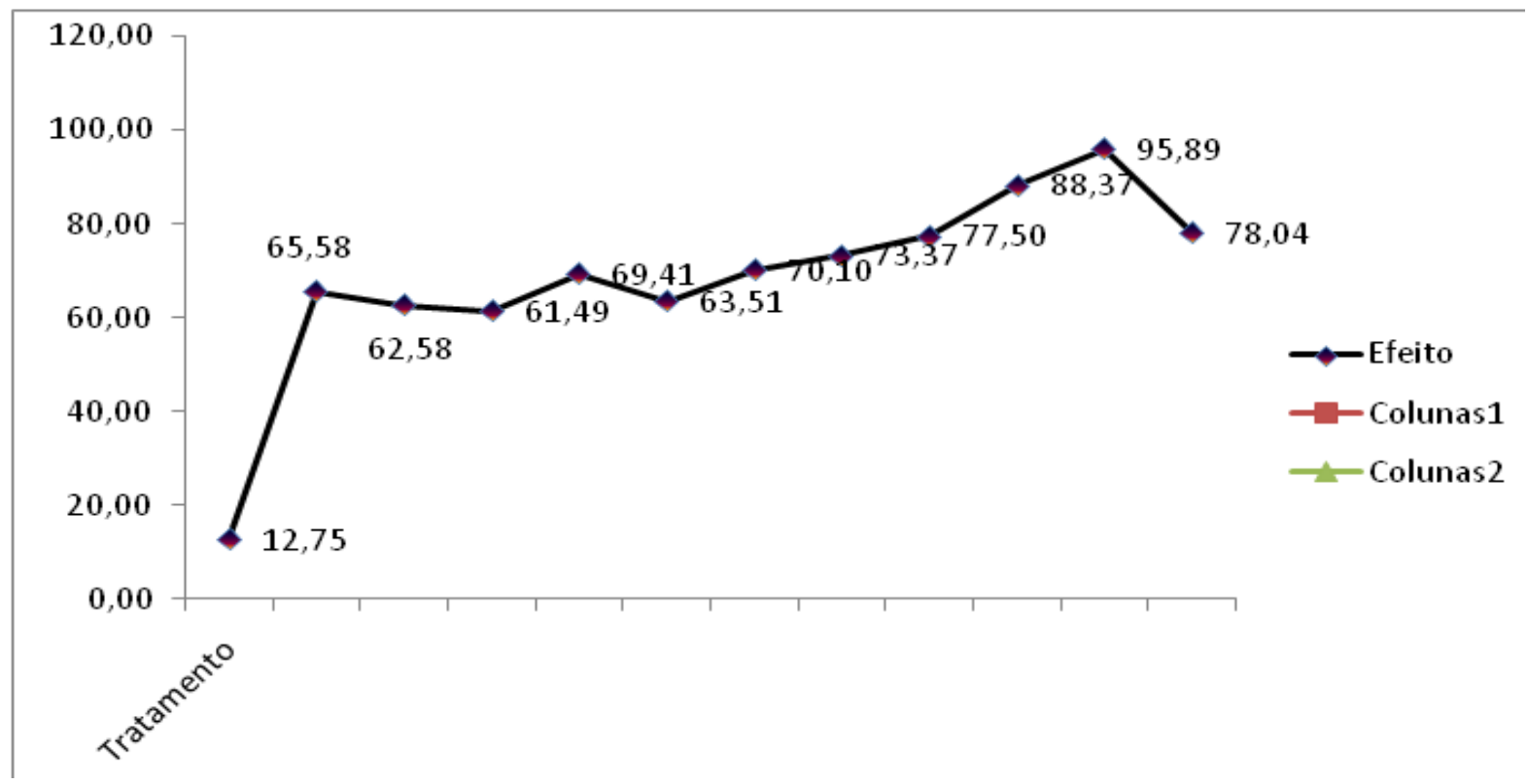

Fig. 1 - Gráfico demonstrativo dos diferentes resultados na evolução das concentrações testadas do EHA de L. sidoides. 


\section{REFERÊNCIAS}

ALCÂNTARA, JUNIOR, J.P.; AYALA-OSUNA, J.T.; QUEIROZ, S.R.O.D.; RIOS, A.P. Levantamento etnobotânico e etnofarmacológico de plantas medicinais do município de Itaberaba-BA para cultivo e preservação. Sitientibus. Série Ciências Biológicas, v.5, n.1, p.39-44, 2005.

ALMEIDA, M.A. O. BOTURA, M.B.; SANTOS, M.M. dos; ALMEIDA, G.N.; DOMINGUES, L.F.; COSTA, S.L.; BATATINHA, M.J.M. Efeitos dos extratos aquosos de folhas de Cymbopogon citratos (DC.) staf (capim-santo) e de Digitaria insulares (I) fedde (Capim-açu) sobre cultivo de larvas de nematóides gastrintestinais de caprinos. Revista Brasileira de Parasitologia Veterinária, v.12, n.3, p.125-129, 2003.

AROSEMENA, N.A.E.; BEVILAQUA, C.M.L.; MELO, A.C.F.L.; GIRÃO, M.D. Seasonal variations of gastrointestinal nematodes in sheep and goats from semi-arid área in Brazil. Revista Medicina Veterinária, v.150,. p.1114, 1998.

BEVILAQUA, C.M.L.; VASCONCELOS, A.L.F.; MORAIS, S.M. Ovicidal and larvicidal activity of Lippia sidoides and Ocimum gratissimum essencial oils against Haemonchus contortus. In: INTERNATIONAL CONFERENCE OF THE WORLD ASSOCIATION FOR THE ADVANCEMENT OF VETERINARY PARASITOLOGY, 20., 2005, Christtchurch, NZ. Session D.

CHARLES, T.P. Seasonal prevalence of gastrointestinal nematodes of goats in Pernambuco State, Brazil. Veterinary Parasitology, v.30, p.335-343, 1989.

FARIAS, M.P.O. Avaliação "in vitro" da atividade ectoparasiticida e anti-helmíntica da andiroba (Carapa guianenses Aubli.). 2007. 134f Dissertação (Mestrado em Ciência Veterinária) - Universidade Federal Rural de Pernambuco, Departamento de Medicina Veterinária, Recife, 2007.

MERCOSUL. Grupo Mercado Comum. Regulamento técnico para registros de produtos antiparasitários de uso veterinário. Decisão $\mathrm{N}^{\circ} 4 / 91$, Resolução $\mathrm{N}^{\circ} 11 / 93$. MERCOSUL, Resolução Nº 76., 1996.

HAMMOND, J.A.; FIELDING, D.; BISHOP, S.C. Prospects for plant anthelmintics in veterinary medicine. Veterinary Research Communications, v.21, p.213-228, 1997

HERD, R. Impactos ambientais associados aos compostos endectocidas. In: PADILHA, T. (Ed.). Controle dos nematóides gastrintestinais em ruminantes. Coronel Pacheco: EMBRAPA - CNPGL, 1996. p.95-111.

HOUNZANGBE-ADOTE, M.S.; PAOLINI, V.; FOURASTE, I.; MONTAIROU, K.; HOST, H. in vitro effects of four tropical plants on three life-cycle stages of the parasitic nematode, Haemonchus contortus. Research in Veterinary Science, v.78, p.155-160, 2005.
LACOSTE, E.; CHAUMONT, J.P.; MANDIN, D.; PLUMEL, M.M.; MATOS, F.J.A. Antiseptic properties of essential oil of Lippia sidoides Cham.; application to the cutaneous microflora. Annales Pharmaceutiques Françaises, v.54, p.228-230, 1996.

LEMOS, T.L.G.; MATOS, F.J.A.; ALENCAR, J.W.; CRAVEIRO, A.A.; CLARK, A.M.; MCCHESNEY, J.D. Antimicrobial activity of essential oils of Brazilian plants. Phytotherapy Research, v.4, n.2, p.82-84, 1990.

LORENZI, H.; MATOS, F.J.A. Plantas medicinais no Brasil: nativas e exóticas. Nova Odessa: Instituto Plantarum, 2002. 512p.

MACAMBIRA, L.M.A.; ANDRADE, C.H.S.; MATOS, F.J.A.; CRAVEIRO, A.A. Naphtoquinoids from Lippia sidoides. Journal of Natural Products, v.49, p.310-312, 1986.

MATOS, F.J.A.; MACHADO, M.I.L.; CRAVEIRO, A.A.; ALENCAR, J.W.; SILVA, M.G. Medicinal plants of Northeast Brazil containing thymol and carvacrol Lippia sidoides Cham. and L. gracillis H.B.K. (Verbenaceae). Journal of Essencial Oil Research, v.11, n.6, p.666-668, 2000.

PINHEIRO, R.R.; GOUVEIA, A.M.G.; ALVES, F.S.F.; HADDAD, J.P.A. Aspectos epidemiológicos da caprinocultura cearense. Arquivo Brasileiro de Medicina Veterinária e Zootecnia, v.52, p.534-543, 2000.

POWER, K.G. et al. World Association for the Advanement of Verinary Parasitoy(W.A.A.V.P.) - Guidelines for evaluating the efficacy of anthelmintics in ruminants (bovine and ovine).Veterinary Parasitolgy, v.10, p.265268, 1982.

STEPHENS, M.A. EDF statistics for goodness of fit and some comparisons. Journal of the American Statistical Association, v.69, p.730-737, 1974.

TERBLANCHÉ, F.C.; KORNELIUS, G. Essencial oil constituents of the genus Lippia (Verbenaceae) - A literature review. Journal of Essencial Oil Research, n.8, 471-485,1996.

UENO, H,; GONÇALVES, P.C. Manual para diagnóstico das helmintoses de ruminantes. 3.ed. Tokyo:

International Cooperation Agency. Porto Alege: Faculdade de Veterinária da UFRGS, 1998. 166p.

VIEIRA, L. da S.; CAVALCANTE, A.C.R. Resistência anti-helmíntica em rebanhos caprinos no Estado do Ceará. Pesquisa Veterinária Brasileira, v.19, n.3/4, p.99103, 1999.

Recebido em 24/4/08

Aceito em 17/1/11 